\title{
Quantum Griffiths singularities in TiO superconducting thin films with insulating normal states
}

\author{
Chao Zhang ${ }^{1}$, Yunjie Fan', Qiaoling Chen', Tianyi Wang ${ }^{1,2}$, Xiang Liu', Qi Li ${ }^{2}$ Yuewei Yin $\mathbb{D}^{1}$ and Xiaoguang Li,3,4
}

\begin{abstract}
A superconductor-metal transition (SMT) with an unconventional diverging dynamic critical exponent was recently discovered, and it drew tremendous attention because this signature of a quantum Griffiths singularity (QGS) was thought to be a common characteristic of low-disorder crystalline superconductors. However, because the QGS was observed only in limited materials with metallic normal states, the question of whether the QGS exists in other superconducting systems is still unanswered. In this paper, a superconductor-insulator transition (SIT) is observed in $\mathrm{TiO}$ thin films with insulating normal states, which offers a more universal platform for investigating the QGS. A thickness-tuned SIT is obtained when the magnetic field is zero. Importantly, a magnetic field-tuned SIT with a diverging dynamic critical exponent, which is direct evidence of a QGS, is observed in TiO thin films with different thicknesses. By constructing a comprehensive phase diagram, it is demonstrated that the critical magnetic field $H_{c}$ tends to saturate as the temperature approaches $0 \mathrm{~K}$, which is different from the upturn trend of $H_{c}$ observed in SMT systems and probably due to the weaker Josephson coupling of the locally ordered superconducting islands (rare regions) in a weakly insulating normal state background. The results extend the QGS scenario from only SMT systems to SIT systems, and they provide vital evidence that QGSs are common in crystalline superconducting thin films, which has possible applications in quantum-computing devices.
\end{abstract}

\section{Introduction}

The superconductor-insulator (metal) transition (SIT/ $\mathrm{SMT}$ ), a remarkable paradigm of the quantum phase transition (QPT), has been an important issue because this exotic phenomenon not only extends our understanding of the novel physical nature of the QPT but also contributes to the design of quantum devices, such as superconducting qubits ${ }^{1,2}$. The ground state of SIT/SMT can be tuned by a variety of external parameters, such as the magnetic field ${ }^{3}$, thickness ${ }^{4,5}$, disorder ${ }^{6}$, chemical

\footnotetext{
Correspondence: Yuewei Yin (yyw@ustc.edu.cn) or Xiaoguang Li (lixg@ustc. edu.cn)

${ }^{1}$ Hefei National Laboratory for Physical Sciences at the Microscale and CAS Key Laboratory of Strongly-Coupled Quantum Matter Physics, Department of Physics, University of Science and Technology of China, 230026 Hefei, China ${ }^{2}$ Department of Physics, Pennsylvania State University, University Park, PA, USA Full list of author information is available at the end of the article.

These authors contributed equally: Chao Zhang, Yunjie Fan
}

composition $^{7}$, charge density ${ }^{8}$, or gate voltage ${ }^{9,10}$, instead of thermal fluctuations. The SIT/SMT phenomena were initially studied in granular or amorphous thin films, which are highly disordered, and recently, studies of the phenomena have expanded to crystalline material systems ${ }^{11}$. Despite the efforts over the past several decades, many issues still remain. For example, even though a socalled 'dirty-boson' model has been commonly used, different universality classes defined by different critical exponents and different numbers of quantum critical points (QCPs) have been observed ${ }^{12-14}$.

In 2015, a totally different type of SMT called a quantum Griffiths singularity (QGS) was strikingly reported in a molecular-beam-epitaxy (MBE)-grown Ga trilaye ${ }^{14}$ that shows diverging dynamic exponents and a continuous line of QCPs. Since this discovery, examples of SMTs with a QGS have been observed in a $\mathrm{NbSe}_{2}$ monolayer $^{15}$, a

\section{(c) The Author(s) 2019}

(c) Open Access This article is licensed under a Creative Commons Attribution 4.0 International License, which permits use, sharing, adaptation, distribution and reproduction cc) in any medium or format, as long as you give appropriate credit to the original author(s) and the source, provide a link to the Creative Commons license, and indicate if changes were made. The images or other third party material in this article are included in the article's Creative Commons license, unless indicated otherwise in a credit line to the material. If material is not included in the article's Creative Commons license and your intended use is not permitted by statutory regulation or exceeds the permitted use, you will need to obtain permission directly from the copyright holder. To view a copy of this license, visit http://creativecommons.org/licenses/by/4.0/. 
$\mathrm{LaAlO}_{3} / \mathrm{SrTiO}_{3}$ (110) interface ${ }^{16}$, quasi-one-dimensional $\mathrm{Ta}_{2} \mathrm{PdS}_{5}$ nanowires ${ }^{1}$, and ion-gated superconducting surfaces of $\mathrm{ZrNCl}$ and $\mathrm{MoS}_{2}$ single crystals ${ }^{17}$. In the QGS picture, locally ordered superconducting islands (rare regions) that survive in the normal state background exist. The QCPs and their dynamic behaviors are strongly influenced by these rare regions. Compared to the conventional SIT/SMT picture, such as the 'dirty-boson' model $^{18}$, in which the universality class of a phase transition determines the critical behaviors, the quenched disorders (i.e., grain boundaries, dislocations, impurities, etc.) can have profound effects on the QGS. The QGSactivated scaling will replace the traditional power-law scaling when coarse-grained disorder strength diverges, and QCPs of infinite randomness will show up. In this case, the QPT can be characterized by a diverging critical exponent towards the QCPs ${ }^{19}$.

Although the QGS was observed only in limited SMT materials with metallic normal states, a claim was made that it was a generic characteristic of low-disorder crystalline thin films ${ }^{11}$. Therefore, it is necessary to explore the QGS in other thin film systems to better understand the QGS phenomenon. It will be especially interesting to examine SIT systems with semiconducting or insulating normal states in which the critical correlations of superconducting rare regions are much weaker. Recently, interest in superconducting titanium oxides has resurged, and superconductivities in structure-selected $\mathrm{Ti}_{2} \mathrm{O}_{3}{ }^{20}$, nano-TiO crystals ${ }^{21}, \gamma-\mathrm{Ti}_{3} \mathrm{O}_{5}$, and $\mathrm{Ti}_{4} \mathrm{O}_{7}{ }^{22}$ have been reported. Crystalline superconducting titanium oxide thin films are good systems for studying the QPT. In previous studies, we succeeded in growing crystalline $\mathrm{TiO}$ thin films on $\alpha-\mathrm{Al}_{2} \mathrm{O}_{3}$ (0001) substrates ${ }^{23,24}$, and we found an oxygen-content-tuned SIT in $\sim 80 \mathrm{~nm} \mathrm{TiO}_{x}$ films, but no QGS was observed because of the narrow range of measured temperatures ${ }^{25}$. In the present paper, we grow crystalline $\mathrm{TiO}$ epitaxial thin films with different thicknesses. As the thickness decreases, an SIT appears, and its critical resistance is approximately $h / 4 e^{2}=6.45 \mathrm{k} \Omega / \square$. Furthermore, by investigating the SIT driven by a perpendicular magnetic field, the QGS is revealed to have a diverging critical exponent product $z v$. Finally, the phase diagram of the SIT with a QGS is constructed with a distinct quantum Griffiths state and is compared with that of previous SMT systems, which indicates the important role of the normal state background.

\section{Materials and methods}

\section{Sample preparation}

By using the pulsed laser deposition (PLD) technique, epitaxial $\mathrm{TiO}$ thin films of various thicknesses were grown on commercial (0001) $\alpha-\mathrm{Al}_{2} \mathrm{O}_{3}$ substrates. The $\mathrm{Ti}_{2} \mathrm{O}_{3}$ target was ablated by an excimer laser (Coherent COMPexPro205, $\lambda=248 \mathrm{~nm}$ ). The pressure of the chamber, deposition temperature, laser energy density, and target-substrate distance used for the deposition were $2 \times 10^{-4} \mathrm{~Pa}, 850^{\circ} \mathrm{C}, 2.0 \mathrm{~J} \mathrm{~cm}^{-2}$, and $4.5 \mathrm{~cm}$, respectively.

\section{Structural characterizations}

X-ray diffraction (XRD) characterizations were carried out by a commercial X-ray diffractometer (Panalytical X'pert, $\sim 1.5406 \AA$ ). The thicknesses and surface morphologies were measured using a scanning electron microscope (SEM, Hiachi UHR FE-SEM SU8220) and an atomic force microscope (AFM, Oxford Instruments MFP-3D Infinity). For the structure and chemical composition characterizations, the high-angle annular darkfield (HAADF) scanning transmission electron microscopy (STEM) images and core-level electron energy-loss spectroscopy (EELS) images were measured by using a transmission electron microscope with a spherical aberration corrector (Talos F200X).

\section{Electrical transport measurements}

For the Hall resistance and transport measurements, a Hall bar (length $(L)$ of $\sim 400 \mu \mathrm{m}$ and width (W) of $\sim 30 \mu \mathrm{m}$ ) was prepared. The transport measurements in the magnetic field were investigated by a four-probe method in a Physical Property Measurement System (Quantum Design) equipped with a ${ }^{3} \mathrm{He}$ refrigerator, and a dilution refrigerator down to $0.05 \mathrm{~K}$ was used to measure some of the resistances in the zero magnetic field. Sheet resistances were calculated by $R_{\mathrm{s}}=R W / L$. The voltage-current $(V-I)$ characteristics were tested using a current source and a nanovoltmeter (Keithley 6221 and 2182A).

\section{Results}

\section{Structural and chemical characterizations}

Figure 1a shows the XRD $(\theta-2 \theta)$ scans of $\mathrm{TiO}$ epitaxial thin films of different thicknesses deposited on (0001) sapphire substrates. For these $\mathrm{TiO}$ thin films with different thicknesses, only the diffraction peaks of $\alpha-\mathrm{Al}_{2} \mathrm{O}_{3}$ (0006) and $\mathrm{TiO}(111)$ and (222) were observed ${ }^{24}$. As the film thickness decreases, the full width at half maximum (FWHM) of the TiO (111) $\theta-2 \theta$ peaks increases, while the FWHM of the TiO (111) rocking curves decreases because the surface roughness decreases (Supplementary Fig. S1). The lower roughness in the thinner film is also confirmed by the AFM measurements (Supplementary Fig. S2 and Table S1). In this study, the thicknesses were determined by cross-sectional SEM (Supplementary Fig. S2).

The HAADF-STEM image of the representing $80 \mathrm{~nm}$ sample indicates that the $[111] /[112]$ crystalline orientations of the $\mathrm{TiO}$ thin film are parallel to the $[0001] /[11 \overline{2} 0]$ axes of the $\alpha-\mathrm{Al}_{2} \mathrm{O}_{3}$ substrate, as shown in Fig. 1b. Furthermore, more comprehensive HAADF-STEM studies at different areas indicate the existence of grain boundaries 

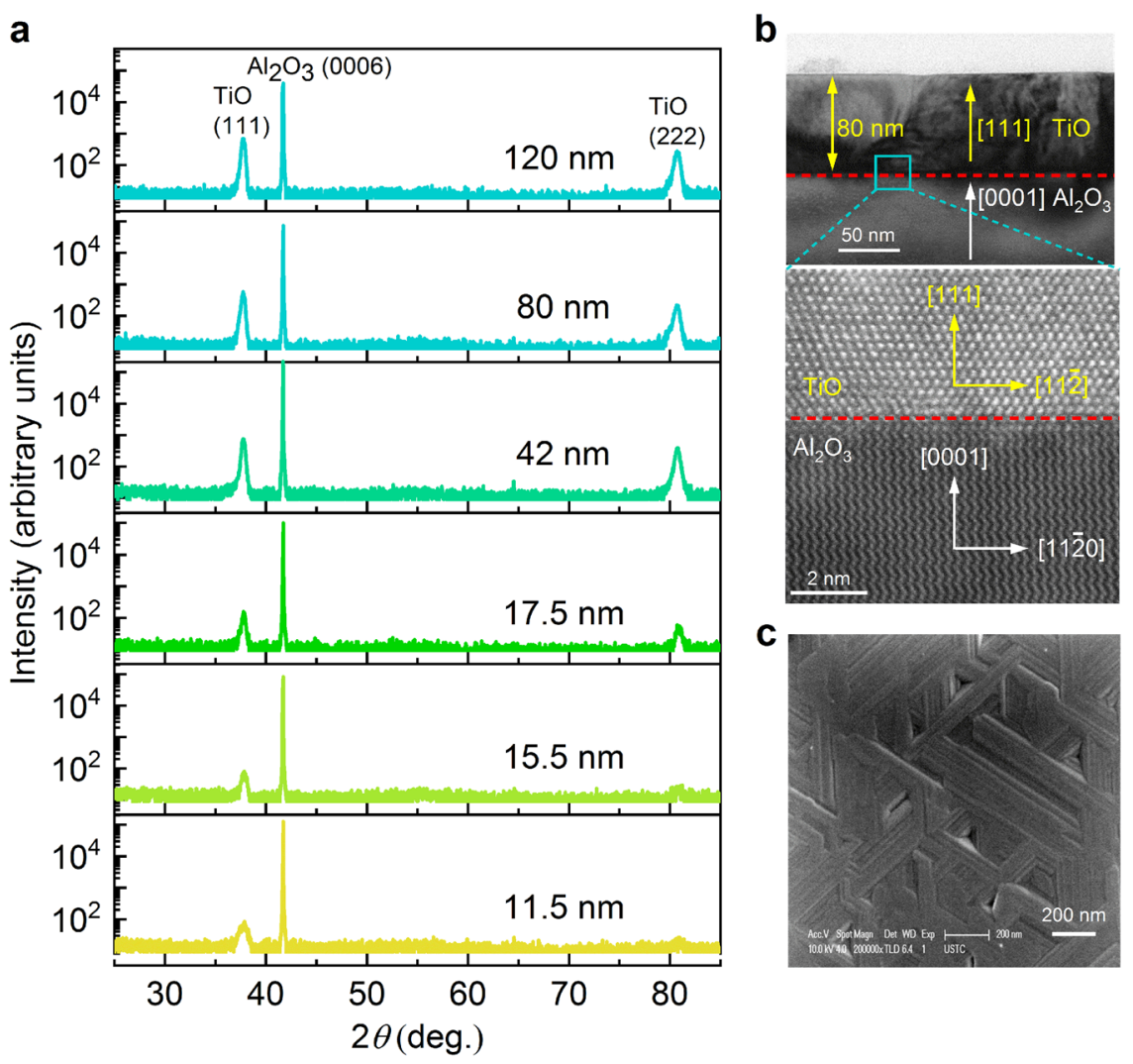

Fig. 1 Structural characterizations of the TiO thin films. a $\theta / 2 \theta$ XRD patterns of the TiO/ $a-\mathrm{Al}_{2} \mathrm{O}_{3}(0001)$ heterostructures for different thicknesses. b Cross-sectional TEM and HAADF-STEM images of a TiO thin film viewed along the [1100] direction of $a-\mathrm{Al}_{2} \mathrm{O}_{3}$. c Surface morphology image measured by SEM.

(Supplementary Fig. S3). This phenomenon occurs because some areas of the $\mathrm{TiO}$ present a different crystalline orientation, which can be converted to that shown in Fig. 1 b by a rotation of $60^{\circ}$ (or integral multiple of $60^{\circ}$ ) around the [111] axis of the TiO thin film. The surface morphology image obtained by SEM is shown in Fig. 1c. It is noteworthy that triangular facets were observed on the surface, which reflects the more stable (100) facets of the rock-salt type $\mathrm{TiO}$ deposited on the hexagonal $\alpha-\mathrm{Al}_{2} \mathrm{O}_{3}$ substrate 22,26 . The different orientations of the triangular facets may also reflect the structural features with grain boundaries (see detailed discussions in Supplementary S1). In addition, quantitative EELS analyses were performed to estimate the compositions of $\mathrm{TiO}$ thin films with different thicknesses ${ }^{27}$, as shown in Supplementary Figs. S4-S6. Their average O/Ti ratios are all $\sim 1.14$, which indicates that the oxygen contents for these samples are identical.

\section{Thickness-tuned SIT in a zero magnetic field}

The temperature dependences of sheet resistances $R_{\mathrm{s}}(T)$ in a zero magnetic field for $\mathrm{TiO}$ epitaxial thin films with different thicknesses are shown in Fig. 2a. The inset of
Fig. 2a displays an enlarged view of the sheet resistive transitions at low temperatures. The superconducting thin films with thicknesses between 15.5 and $120 \mathrm{~nm}$ show an abrupt drop in the resistance to zero at the critical temperatures, and no evidence of the re-entrant behavior ${ }^{28}$ was found. A further reduction in the film thickness to $11.5 \mathrm{~nm}$ leads to a negative slope in $\mathrm{d} R_{\mathrm{s}}(T) /$ $\mathrm{d} T$ when the temperature approaches zero, which indicates its insulating nature. These results imply that a clear SIT appears in TiO thin films tuned by thickness. As illustrated in the inset of Fig. 2a, the critical transition sheet resistance is $\sim R_{\mathrm{Q}}=6.45 \mathrm{k} \Omega / \square$, which is in agreement with the quantum resistance for pairs ${ }^{10}$. Similar SIT behaviors has been achieved by tuning the oxygen content in $\mathrm{TiO}_{x}$ thin films ${ }^{25}$; the ability to tune SITs by thickness and/or composition has been observed in other materi$\mathrm{als}^{4,29}$ as well. Figure $2 \mathrm{~b}$ shows that the value of $T_{\mathrm{c}}$ decreases as the thickness of the $\mathrm{TiO}$ thin films decreases, where $T_{\mathrm{c}}$ is defined as the temperature corresponding to $90 \%$ of the sheet resistance in a normal state. This result occurs because as the film thickness decreases, the scattering, which may reflect the disorder strength, increases due to the larger proportions of the surface and interface 

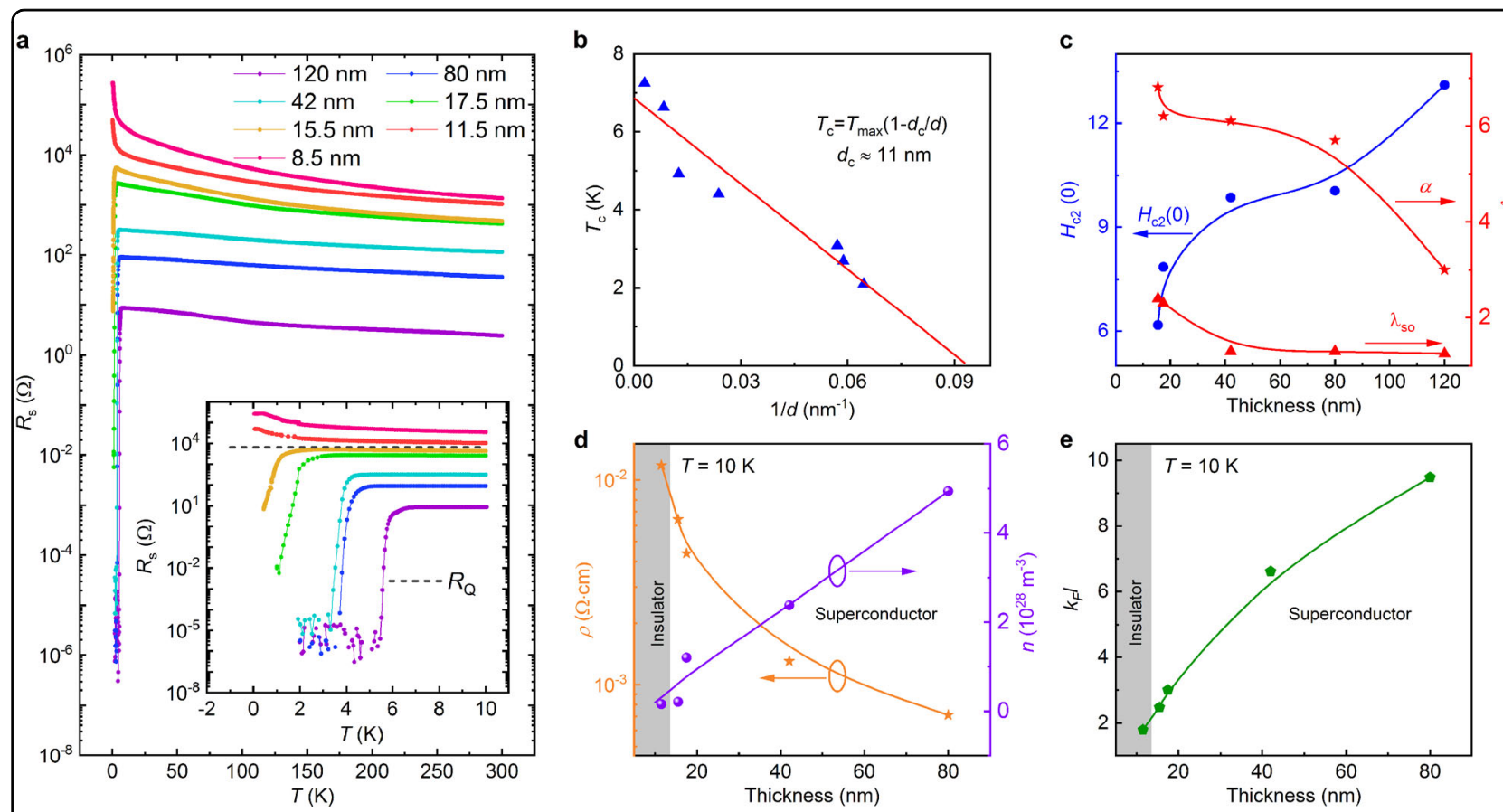

Fig. 2 Thickness-tuned SIT and carrier density of TiO thin films. a Temperature dependences of sheet resistances for TiO thin films with various thicknesses. The inset shows an enlarged view of sheet resistances at low temperatures and the critical quantum resistance $R_{\mathrm{Q}}=6.45 \mathrm{k} \Omega / \square \cdot \mathbf{b}$ Variation of $T_{c}$ with inverse film thickness $d$. The solid line is the fitting result by Simonin's model $\left(T_{c}=T_{\max }\left(1-d_{c} / d\right)\right)^{32}$. c Thickness-dependent $H_{c 2}(0)$ and fitting parameters ( $a$ and $\lambda_{\text {so }}$ ) of WHH theory. $\mathbf{d}$ Variations of resistivity and carrier concentration with thickness of TiO thin films. e Thickness dependence of $k_{F} /$ for $\mathrm{TiO}$ thin films.

areas in the thinner film ${ }^{30,31}$. When the film thickness approaches a critical level, the system is no longer in a superconducting state ${ }^{4}$. By inserting a surface-energy term into the Ginzburg-Landau equations, Simonin proposed a relationship between $T_{\mathrm{c}}$ and film thickness $d$ as follows ${ }^{32}$ :

$$
T_{\mathrm{c}}=T_{\max }\left(1-d_{\mathrm{c}} / d\right) .
$$

Here, $d_{\mathrm{c}}$ is the critical thickness below which the superconductivity disappears, and $T_{\max }$ is a fitting parameter that corresponds to the maximum $T_{\mathrm{c}}$. The origin of this surface term is related to the parameters affected by the disorders, such as the mean free path. As shown in Fig. 2b, Eq. (1) fits our results well with parameters $T_{\max }=6.75 \mathrm{~K}$ and $d_{\mathrm{c}}=11 \mathrm{~nm}$, and $d_{\mathrm{c}}$ is close to the experimental critical thickness of $\mathrm{TiO}$ of $\sim 11.5 \mathrm{~nm}$. It should be noted that $d_{\mathrm{c}}$ is material dependent. For example, $d_{c}$ is $1.46 \mathrm{~nm}$ for $\mathrm{Nb}_{x} \mathrm{Si}_{1-x} 33,1.56 \mathrm{~nm}$ for $\mathrm{YBa}_{2} \mathrm{Cu}_{3} \mathrm{O}_{y}$ ultrathin films ${ }^{34}$ and $30 \mathrm{~nm}$ for $\mathrm{FeSe}_{0 \cdot 3} \mathrm{Te}_{0.7}{ }^{35}$. A recent work ${ }^{20}$ on the superconductivity of orthorhombic $\mathrm{Ti}_{2} \mathrm{O}_{3}$ demonstrates a $d_{\mathrm{c}}$ of $\sim 10 \mathrm{~nm}$, which is similar to the $\mathrm{TiO}$ thin films in this study.

From the $R_{\mathrm{s}}-T$ curves in various magnetic fields, we can obtain the temperature-dependent upper critical field $H_{\mathrm{c} 2}$, which is defined as the field corresponding to $90 \%$ of the normal state resistivity, as shown in Fig. S7. The
Werthamer-Helfand-Hohenberg (WHH) model ${ }^{36}$ can be used to describe the $H_{\mathrm{c} 2}(T)$ behavior (see detailed discussions in Supplementary S2). The thickness-dependent $H_{\mathrm{c} 2}(0)$ and fitting parameters (relative strength of spin and orbital effect parameter $\alpha$ and spin-orbit scattering constant $\lambda_{\text {so }}$ ) of WHH theory are summarized in Fig. 2c. Superconductivity is suppressed via the orbital and Pauli spin-paramagnetic effects in the external magnetic fields, and the spin-orbit scattering effect should be taken into account. The fitting parameters $\alpha$ and $\lambda_{\text {so }}$ increase as the thickness decreases, which implies enhancements of the spin-paramagnetic effect and spin-orbit scattering effect, respectively, that may be caused by the enhanced disorder strength $^{37,38}$.

In general, the carrier density, disorder strength, and electron localization length are important factors that affect superconductivity. To obtain the carrier density, the Hall resistances $R_{x y}(H)$ versus magnetic fields of $\mathrm{TiO}$ thin films are investigated at 300 and $10 \mathrm{~K}$ (Supplementary Fig. S8). According to the Hall measurements, dominating $n$-type electronic charge carriers are confirmed, and the carrier density decreases as the film thickness decreases, as shown in Fig. 2d. Suppression of the carrier concentration as the thickness decreases has been observed in many different systems ${ }^{39-41}$. This phenomenon may be related to the disorder strength, surface state, band gap 
narrowing effect ${ }^{40,42}$, etc. (see detailed discussions in Supplementary S3). The decrease in carrier concentration would partially suppress the Cooper pair density and thereby the superconductivity transition temperature ${ }^{43}$. To evaluate the disorder strength, it is necessary to analyze the Loffe-Regel parameter $k_{\mathrm{F}} l$, where $k_{\mathrm{F}}$ is the Fermi wave vector and $l$ is the electronic mean free path. A lower $k_{\mathrm{F}} l$ means higher disorder strength, and the disorder scales can be divided into different regimes: strong disorder $\left(k_{\mathrm{F}} l<1\right)$, lower disorder $\left(10>k_{\mathrm{F}} l>1\right)$, and very low disorder $\left(k_{F} l>10\right)$ at a moderately clean limit ${ }^{44}$. The $k_{F} l$ can be determined from the Hall coefficient $R_{\mathrm{H}}$ and resistivity $\rho$ by the formula ${ }^{45}$ :

$$
k_{\mathrm{F}} l=\left(3 \pi^{2}\right)^{2 / 3} \hbar R_{\mathrm{H}}^{1 / 3} / \rho e^{5 / 3}
$$

where $\hbar$ is the reduced Planck's constant and $e$ is the elementary charge. As shown in Fig. 2e, the value of $k_{\mathrm{F}} l$ decreases as the film thickness decreases, which reflects a monotonic increase in the disorder strength. When $k_{\mathrm{F}} l<$ $1.8(d<11.5 \mathrm{~nm})$, the global superconducting ground state is destroyed. To gain deeper insight into the thicknesstuned SIT, the electron localization length $\xi_{1}$ is estimated by analyzing the transport properties of $\mathrm{TiO}$ thin films in normal states using the variable range hopping model ${ }^{46}$ (see Supplementary S4 and Fig. S9). We can see that $\xi_{1}$ decreases as the thickness of the $\mathrm{TiO}$ decreases, which reflects the increase in the disorder strength. In accordance with the suppressed carrier density for thinner $\mathrm{TiO}$ films, $\xi_{1}$ decreases. Both the lower carrier density and smaller $\xi_{1}$ correspond to a weakened superconductivity.

\section{Magnetic-field-tuned SIT with QGS}

Next, we investigate the magnetic-field-tuned SIT with QGS for TiO thin films with different thicknesses. Figure $3 \mathrm{a}, \mathrm{c}$, e shows the temperature-dependent sheet resistances $R_{\mathrm{s}}(T)$ of $42,17.5$, and $15.5 \mathrm{~nm}$ films in different perpendicular magnetic fields. The superconductivity of the $\mathrm{TiO}$ thin film is suppressed in a higher magnetic field. With a sufficiently large magnetic field, the system gradually becomes a weak insulator. Between the superconducting and insulating regimes, there exists a critical magnetic field where the $R_{\mathrm{s}}(T)$ tends to saturate to a constant at a low temperature, as denoted by the arrows in Fig. 3a, c, e. Figure 3b, d, f shows the magnetoresistance isotherms for the $42,17.5$, and $15.5 \mathrm{~nm}$ samples, respectively. For the three samples, each pair of $R_{\mathrm{s}}(H)$ curves cross at different points, and multiple crossing points of the adjacent isotherms, i.e., the SIT "critical" points, form a continuous line, which is a signature of the QGS. The magnetic fields of these crossing points, defined as $H_{\mathrm{c}}$, are plotted as the red triangles in the insets of Fig. 3b, d, f. The onset superconducting transition temperature $T_{\mathrm{c}}^{\text {onset }}$, which represents the emergence of superconductivity, defined as $\mathrm{d} R_{\mathrm{s}}(T) / \mathrm{d} T=0$ in the $R_{\mathrm{s}}(T)$ curves for different magnetic fields, is also extracted (see Supplementary Fig. S10 for details). Interestingly, the curves of $H_{\mathrm{c}}(T)$ and the fields corresponding to $T_{\mathrm{c}}^{\text {onset }}(H)$ have almost the same trace (see the insets of Fig. 3b, d, f because both parameters (i.e., temperature and magnetic field) indicate the onset of superconducting fluctuation ${ }^{17}$.

\section{Discussion}

In the scenario of a QGS, finite-size scaling (FSS) can be used to analyze the electrical transport results and obtain the values of the critical exponents $z v(z$ and $v$ are the dynamic critical exponent and correlation length exponent, respectively) in different temperature ranges for $\mathrm{TiO}$ samples with different thicknesses ${ }^{14,15}$. The details of the FSS analyses are shown in Supplementary S6 and Figs. S11-S16.

The relationships between the magnetic fields and $z v$ values are shown in Fig. $4 \mathrm{a}-\mathrm{c}$ for different $\mathrm{TiO}$ thin film thicknesses. It can be seen that as the temperature decreases, the $z v$ increases for $\mathrm{TiO}$ samples with different thicknesses. In the region of relatively high temperatures, $z v$ rises smoothly as the magnetic field increases, while $z v$ ramps up steeply in the region of low temperature. This behavior occurs due to the enhanced effect of the quenched disorder as the temperature decreases ${ }^{11}$. In principle, the quenched disorder will introduce locally ordered superconducting rare regions on the microscopic level, which can affect the $z v$ value accordingly. We can use the activated scaling law equation $z \nu=C_{1}\left(H_{\mathrm{c}}^{*}-H\right)^{-\nu \psi}$ to fit the $z v(H)$ curves, where $C_{1}$ is a constant, $H_{\mathrm{c}}^{*}$ is the characteristic magnetic field, and $v \approx 1.2$ and $\psi \approx 0.5^{47,48}$ according to the $2 \mathrm{D}$ infinite-randomness critical exponents. The fitting results reasonably agree with the experimental results for the $42,17.5$, and $15.5 \mathrm{~nm}$ samples and indicate infinite-randomness QCPs. It is also shown that the $z v$ value diverges at the $T \sim 0 \mathrm{~K}$ limit when approaching the critical point $H_{\mathrm{c}}^{*}$. As the thickness decreases, $C_{1}$ increases. This result indicates a more severe increase in the $z v$ value as the magnetic field increases close to $H_{\mathrm{c}}^{*}$ and demonstrates that the influence of disorder on the QGS of an SIT can be tuned by the specimen thickness. Although the temperature for the $15.5 \mathrm{~nm}$ sample is not low enough to obtain $z v$ above 1 , it is worth noting that the critical exponent $z v$ is still increasing as the temperature decreases, and a $z v$ value above 1 should be expected at a lower temperature.

Figure $4 \mathrm{~d}$ shows the normalized magnetic field $\left(H / H_{\mathrm{c}}^{*}\right)$ dependence of $z \nu /\left(\mathrm{C}_{1} H_{\mathrm{c}}^{*-0.6}\right)$ values and the fitting results. The experimental results of samples of different thicknesses are perfectly normalized to a single fitting curve, indicating that the quantum critical behaviors for samples of different thicknesses can be described by a unified QGS picture. 

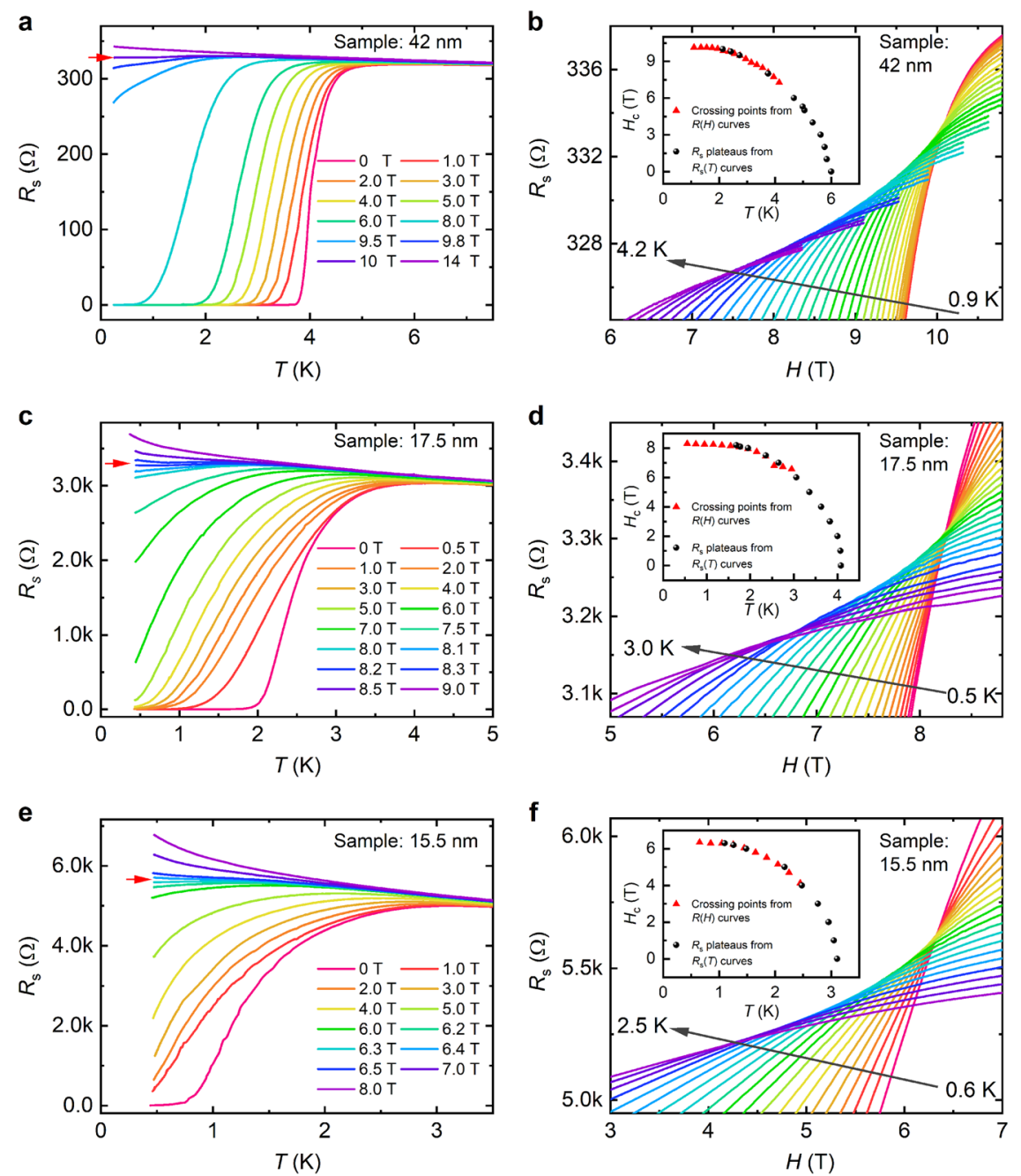

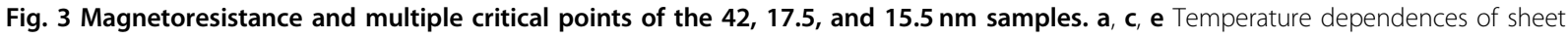
resistances $R_{s}(T)$ for various magnetic fields for the $42,17.5$, and $15.5 \mathrm{~nm}$ samples, respectively. $\mathbf{b}, \mathbf{d}, \mathbf{f}$ Isotherm $R_{5}(H)$ curves measured at different temperatures for the $42,17.5$, and $15.5 \mathrm{~nm}$ samples, respectively. Insets: red triangles: the critical magnetic fields $H_{c}$ extracted from the crossing points of $R_{\mathrm{s}}(H)$ curves at every two adjacent temperatures; and black dots: $T_{\mathrm{c}}^{\text {onset }}$, the temperature plateaus on the $R_{\mathrm{s}}(T)$ curves at different magnetic fields; see Supplementary Fig. S10.

Based on the results of the $\sim 17.5 \mathrm{~nm} \mathrm{TiO}$ epitaxial thin film, a comprehensive phase diagram is proposed for SIT systems with a QGS, as shown in Fig. 5. The comprehensive phase diagram is characterized by $T_{\mathrm{c}}^{\text {onset }}(H)$ (green diamonds), $H_{\mathrm{c}}(T)$ (orange squares), $H_{\mathrm{c} 2}(T)$ (purple hexagons), $H_{\mathrm{c} 2}^{\mathrm{MF}}(T)$ (red circles), and $T_{\text {cross }}(H)$ (cyan triangles). Here, the mean field upper critical field $H_{\mathrm{c} 2}^{\mathrm{MF}}(T)$ is estimated according to the Ullah-Dorsey scaling theory (see Supplementary Fig. S17 for details) ${ }^{49}$, and the crossover temperature $T_{\text {cross }}(H)$ is calculated by the Arrhenius plot analyses of the $R_{\mathrm{s}}(T)$ curves (see details in Supplementary Fig. S18). $H_{\mathrm{c}}(T)$ and $T_{\mathrm{c}}^{\text {onset }}(H)$ represent the onset of superconductivity and separate regions of the weakly insulating normal state and superconducting state. As the temperature decreases, the weakly localized insulator at low magnetic fields crosses over, in turn, the thermal fluctuation region below $H_{\mathrm{c}}(T)$, the thermal phase fluctuation (thermal creep) region below $H_{\mathrm{c} 2}^{\mathrm{MF}}(T)$, and the quantum phase fluctuation (quantum creep) region below $T_{\text {cross }}(H)$. The quantum vortex creep state evolves to a socalled quantum Griffiths state with the QGS above $H_{\mathrm{c} 2}^{\mathrm{MF}}(T)$ and below $H_{\mathrm{c}}(T)^{17}$. In a region with a sufficiently high magnetic field, the $H_{\mathrm{c}}$ of the SIT with a QGS increases slowly at low temperatures and tends to saturate around $H_{\mathrm{c}}^{*}$ as the temperature approaches $0 \mathrm{~K}$, which is different from the upturn trend of $H_{\mathrm{c}}(T)$ observed in SMT systems $^{1,14,15,17}$. This difference between the SIT and SMT systems with a QGS might be related to the different normal state backgrounds for the superconducting rare regions; the normal state background is insulating in the 

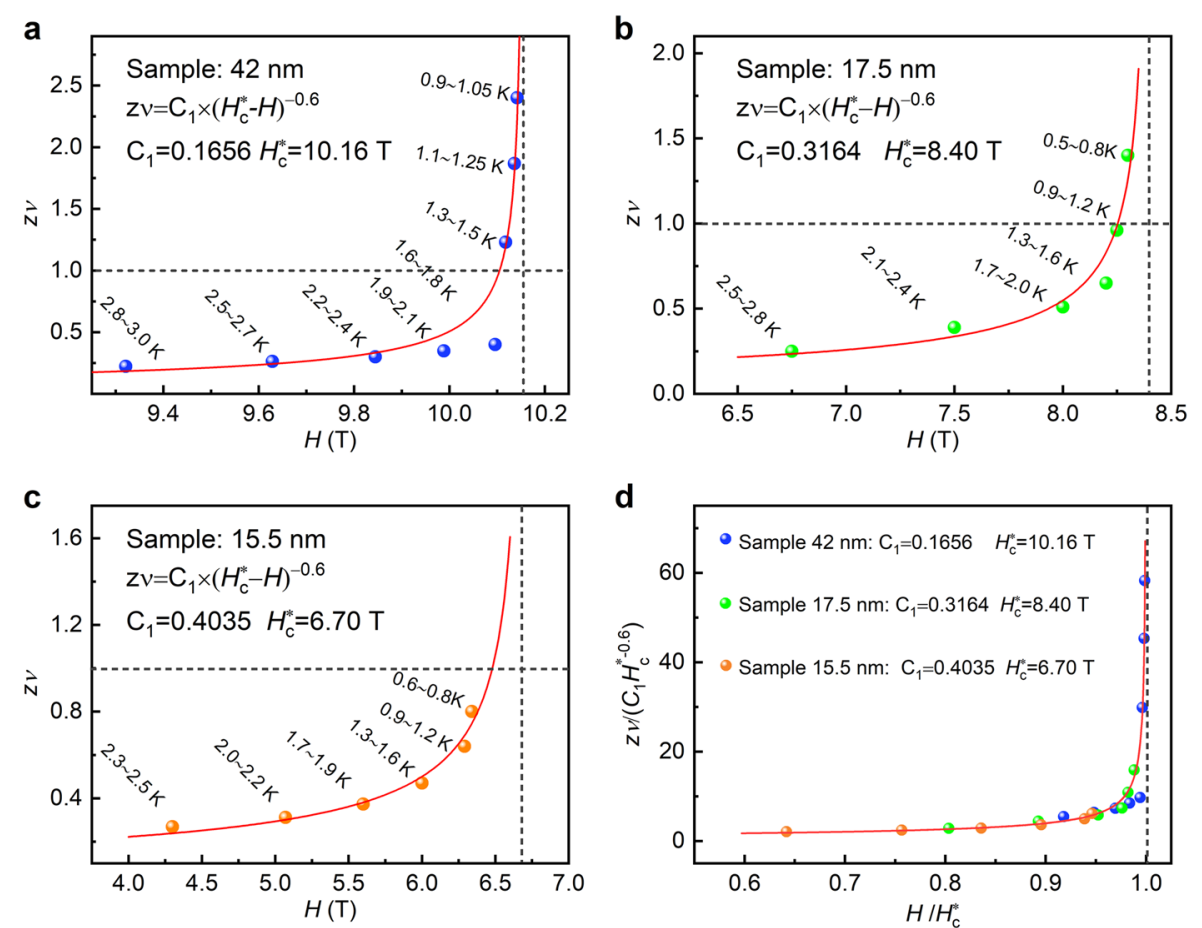

Fig. 4 Activated quantum scaling behavior for TiO thin films. a-c Divergent behavior of critical exponents $z \mathrm{~V}$ in the 42 , 17.5, and 15.5 nm samples, respectively. The $z v$ values are extracted from FSS analyses in different temperature regions. Red lines are the fitting results by $z \nu=\mathrm{C}_{1}\left(H_{\mathrm{c}}^{*}-H\right)^{-0.647}$. The black dashed lines represent constant values with $H=H_{\mathrm{c}}^{*}$ and $z \nu=1$. $\mathbf{d}$ Exponent products $z \nu /\left(\mathrm{C}_{1} H_{\mathrm{c}}^{*-0.6}\right)$ as a function of the normalized magnetic field $H / H_{\mathrm{c}}^{*}$. The black dashed line represents the constant value $H / H_{\mathrm{c}}^{*}$.

TiO SIT system but metallic in the previous SMT systems. As previously discussed, in SMT systems ${ }^{1,11,14,16}$, the QGS in the low-temperature regime is closely related to the locally ordered superconducting rare regions that are coupled through Josephson couplings. The Josephson interaction strength is critically important for the formation and properties of rare regions, and the interaction strength is different for SMT and SIT systems with metallic and insulating normal states, respectively. The insulating background in the SIT system could provide a weaker Josephson coupling than that of the SMT systems, which may affect the quantum Griffiths state region.

It is noteworthy that measuring a sufficiently wide temperature range is important for investigating the QGS with multiple $\mathrm{QCPs}^{16,17}$. Otherwise, the change in the critical exponent $z v$ over a narrow measurement temperature range may be neglected. This is the reason for the absence of the QGS in a previous SIT study of $\mathrm{TiO}_{x}{ }^{25}$. More importantly, in earlier works, the QGS was not observed in any amorphous 2D SMT or SIT superconducting system even with a wide temperature measurement range ${ }^{50}$, which may be related to the high and homogeneous concentration of disorder. The effect of quenched disorders in these homogeneously disordered systems is averaged out under coarse graining, and in this situation, the Harris criterion is not violated ${ }^{11}$, which explains the lack of observations of the QGS. Weakly disordered regions and ordered regions can coexist for crystalline 2D superconductors with weaker disorder strength; therefore, the QGS was observed but only reported in limited SMT systems. Here, although the Josephson couplings of the locally ordered rare regions are weakened by the semiconducting and/or insulating background, the significant QGS of the SIT in crystalline $\mathrm{TiO}$ thin films is still observed, providing important evidence that supports the hypothesis that the QGS is common in crystalline superconductors. Furthermore, the thickness-tuned SIT with a QGS further demonstrates the importance of disorder strength in the superconducting QPT, and the results suggest a method to tune and study the QGS.

In conclusion, we have shown thickness-tuned and magnetic-field-tuned SITs with a QGS in TiO thin films. The film-thickness-tuned superconductivity and SIT are related to the reduction in carrier concentration and enhancement of the localization. By investigating the lowtemperature transport properties of $\mathrm{TiO}$ thin films with different thicknesses, a magnetic-field-induced SIT with a diverging dynamic critical exponent is obtained, which shows a suppressed quantum Griffiths state by the weakly 


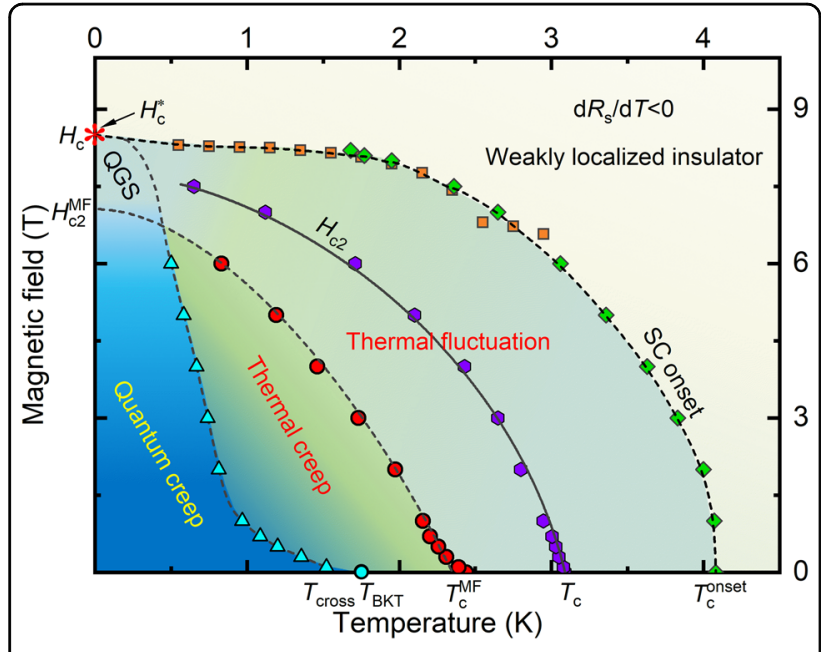

Fig. $5 \mathbf{H}-\boldsymbol{T}$ phase diagram of the TiO SIT system. The plot is based on the experimental data of $\mathrm{TiO} \sim 17.5 \mathrm{~nm}$. Orange squares, green diamonds, purple hexagons, red circles, and cyan triangles represent the crossing points $H_{c}$ of the $R_{s}(H)$ curves in Fig. $3 \mathrm{~d}$ at neighboring temperatures, the superconducting onset $T_{\mathrm{c}}^{\text {onset }}$ (see Supplementary Fig. S10), the upper critical field $H_{c 2}$ (see Supplementary Fig. S7), the mean field upper critical field $H_{\mathrm{c} 2}^{\mathrm{MF}}$ derived from the Ullah-Dorsey (UD) scaling (see Supplementary Fig. S17), and the crossover temperature $T_{\text {cross }}$ (see Supplementary Fig. S18), respectively. The solid line is the fitting result based on the WHH model. The true superconducting state is realized below the

Berezinskii-Kosterlitz-Thouless (BKT) transition temperature ( $T_{\mathrm{BKT}}$ ) (see Supplementary Fig. S19). A thermal fluctuation regime exists between $H_{\mathrm{c} 2}^{\mathrm{MF}}(T)$ and $T_{\mathrm{c}}^{\mathrm{onset}}(T)$. Cyan triangles show the crossover temperature $T_{\text {cross }}(H)$ between the thermal creep regime and the quantum tunneling regime. The system eventually exhibits the quantum Griffiths state up to the characteristic critical magnetic field $H_{\mathrm{c}}^{*}$ (red star).

insulating normal state. The QGS of the SIT in the TiO thin films expands the evidence of the QGS from SMT systems to an SIT system, and the obtained phase diagram of this SIT system with a QGS may be applicable to different crystalline superconducting systems. These results provide considerable insights into the superconducting QPT, which is helpful for designing quantum-computing devices.

\section{Acknowledgements}

This work was supported by the National Natural Science Foundation of China (51790491, 51622209, and 21521001) and the National Key Research and Development Program of China (2016YFA0300103 and 2015CB921201), and the work performed at Penn State University was supported by the United States Department of Energy under Grant no. DE-FG02-08ER46531 (Q.L.). A portion of the sample preparation was performed at the USTC Center for Microscale and Nanoscale Research and Fabrication at the University of Science and Technology of China, Hefei, China.

\section{Author details}

${ }^{1}$ Hefei National Laboratory for Physical Sciences at the Microscale and CAS Key Laboratory of Strongly-Coupled Quantum Matter Physics, Department of Physics, University of Science and Technology of China, 230026 Hefei, China. 2Department of Physics, Pennsylvania State University, University Park, PA, USA. ${ }^{3}$ Key Laboratory of Materials Physics, Institute of Solid State Physics, CAS,
230031 Hefei, China. ${ }^{4}$ Collaborative Innovation Center of Advanced Microstructures, 210093 Nanjing, China

\section{Author contributions}

C.Z. prepared the samples and performed most of the measurements; Y.J.F. and X.L. carried out a part of the transport measurements; Q.L.C. carried out the theoretical calculations; T.Y.W. performed the extremely low temperature measurements in the laboratory of Q.L. at Penn State University; X.G.L. and Y.W.Y. were responsible for the design, planning, and supervision of the overall experiments and calculations; and all authors discussed the data and contributed to the manuscript.

\section{Conflict of interest}

The authors declare that they have no conflict of interest.

\section{Publisher's note}

Springer Nature remains neutral with regard to jurisdictional claims in published maps and institutional affiliations.

Supplementary information is available for this paper at https://doi.org/ 10.1038/s41427-019-0181-3.

Received: 31 January 2019 Revised: 1 October 2019 Accepted: 8 October 2019.

Published online: 20 December 2019

\section{References}

1. Zhang, E. et al. Signature of quantum Griffiths singularity state in a layered quasi-one-dimensional superconductor. Nat. Commun. 9, 4656 (2018).

2. Makise, K., Terai, H., Tominari, Y., Tanaka, S. \& Shinozaki, B. Duality picture of superconductor-insulator transitions on superconducting nanowire. Sci. Rep. 6, 27001 (2016).

3. Hebard, A. \& Paalanen, M. Magnetic-field-tuned superconductor-insulator transition in two-dimensional films. Phys. Rev. Lett. 65, 927 (1990).

4. Haviland, D. B., Liu, Y. \& Goldman, A. M. Onset of superconductivity in the twodimensional limit. Phys. Rev. Lett. 62, 2180 (1989).

5. Zhang, F. et al. Plasma-enhanced pulsed-laser deposition of single-crystalline $\mathrm{Mo}_{2} \mathrm{C}$ ultrathin superconducting films. Phys. Rev. Mater. 1, 034002 (2017).

6. Baturina, T. I., Mironov, A. Y., Vinokur, V. M., Baklanov, M. R. \& Strunk, C. Localized superconductivity in the quantum-critical region of the disorder-driven superconductor-insulator transition in TiN thin films. Phys. Rev. Lett. 99, 257003 (2007).

7. Oh, S., Crane, T. A., Van Harlingen, D. J. \& Eckstein, J. N. Doping controlled superconductor-insulator transition in $\mathrm{Bi}_{2} \mathrm{Sr}_{2-x} \mathrm{La}_{x} \mathrm{CaCu}_{2} \mathrm{O}_{8+\delta}$. Phys. Rev. Lett. 96 107003 (2006).

8. Saito, Y., Kasahara, Y., Ye, J., Iwasa, Y. \& Nojima, T. Metallic ground state in an ion-gated two-dimensional superconductor. Science 350, 409 (2015).

9. Leng, X., Garcia-Barriocanal, J., Bose, S., Lee, Y. \& Goldman, A. M. Electrostatic control of the evolution from a superconducting phase to an insulating phase in ultrathin $\mathrm{YBa}_{2} \mathrm{CaCu}_{3} \mathrm{O}_{7-x}$ films. Phys. Rev. Lett. 107, 027001 (2011).

10. Bollinger, A. T. et al. Superconductor-insulator transition in $\mathrm{La}_{2-x} \mathrm{Sr}_{x} \mathrm{CuO}_{4}$ at the pair quantum resistance. Nature 472, 458 (2011).

11. Saito, Y., Nojima, T. \& Iwasa, Y. Highly crystalline 2D superconductors. Nat. Rev. Mater. 2, 16094 (2016).

12. Markovic, N. Randomness rules. Science 350, 509 (2015).

13. Sun, $Y$. et al. Double quantum criticality in superconducting tin arraysgraphene hybrid. Nat. Commun. 9, 2159 (2018).

14. Xing, Y. et al. Quantum Griffiths singularity of superconductor-metal transition in Ga thin films. Science 350, 542 (2015).

15. Xing, $Y$. et al. Ising superconductivity and quantum phase transition in macrosize monolayer $\mathrm{NbSe}_{2}$. Nano Lett. 17, 6802 (2017).

16. Shen, $\mathrm{S}$. et al. Observation of quantum Griffiths singularity and ferromagnetism at the superconducting $\mathrm{LaAlO}_{3} / \mathrm{SrTiO}_{3}(110)$ interface. Phys. Rev. B 94, 144517 (2016).

17. Saito, Y., Nojima, T. \& Iwasa, Y. Quantum phase transitions in highly crystalline two-dimensional superconductors. Nat. Commun. 9, 778 (2018).

18. Fisher, M. P. Quantum phase transitions in disordered two-dimensional superconductors. Phys. Rev. Lett. 65, 923 (1990). 
19. Harris, A. B. Effect of random defects on the critical behaviour of Ising models. J. Phys. C: Solid State Phys. 7, 1671 (1974).

20. $\mathrm{Li}, \mathrm{Y}$. et al. Observation of superconductivity in structure-selected $\mathrm{Ti}_{2} \mathrm{O}_{3}$ thin films. NPG Asia Mater. 10, 522 (2018).

21. $\mathrm{Xu}$, J. et al. Nano titanium monoxide crystals and unusual superconductivity at 11 K. Adv. Mater. 30, 1706240 (2018).

22. Yoshimatsu, K., Sakata, O. \& Ohtomo, A. Superconductivity in $\mathrm{Ti}_{4} \mathrm{O}_{7}$ and $\gamma-\mathrm{Ti}_{3} \mathrm{O}_{5}$ films. Sci. Rep. 7, 12544 (2017).

23. Zhang, C. et al. Quasi-two-dimensional vortex glass transition and the critical current density in TiO epitaxial thin films. Supercond. Sci. Technol. 31, 015016 (2018).

24. Zhang, C. et al. Enhanced superconductivity in TiO epitaxial thin films. npj Quantum Mater. 2, 2 (2017).

25. Fan, Y. J. et al. Quantum superconductor-insulator transition in titanium monoxide thin films with a wide range of oxygen contents. Phys. Rev. B 98 064501 (2018).

26. Kurokawa, H., Yoshimatsu, K., Sakata, O. \& Ohtomo, A. Effects of phase fraction on superconductivity of low-valence eutectic titanate films. J. Appl. Phys. 122, 055302 (2017).

27. Egerton, R. F. Electron Energy-loss Spectroscopy in the Electron Microscope 3rd edn (Springer Science \& Business Media, LLC, New York, NY, USA, 2011).

28. Jaeger, H. M., Haviland, D. B., Orr, B. G. \& Goldman, A. M. Onset of superconductivity in ultrathin granular metal films. Phys. Rev. B 40, 182 (1989).

29. Breznay, N. P., Tendulkar, M., Zhang, L., Lee, S.-C. \& Kapitulnik, A. Superconductor to weak-insulator transitions in disordered tantalum nitride films. Phys. Rev. B 96, 134522 (2017).

30. Özer, M. M., Jia, Y., Zhang, Z., Thompson, J. R. \& Weitering, H. H. Tuning the quantum stability and superconductivity of ultrathin metal Alloys. Science $\mathbf{3 1 6}$, 1594 (2007)

31. Wu, W., Brongersma, S. H., Hove, M. V. \& Maex, K. Influence of surface and grain-boundary scattering on the resistivity of copper in reduced dimensions. Appl. Phys. Lett. 84, 2838 (2004).

32. Simonin, J. Surface term in the superconductive Ginzburg-Landau free energy: application to thin films. Phys. Rev. B 33, 7830 (1986).

33. Crauste, O., Couedo, F., Berge, L., Marrache-Kikuchi, C. A. \& Dumoulin, L. Destruction of superconductivity in disordered materials: a dimensional crossover. Phys. Rev. B 90, 060203 (2014).

34. Tang, W. H., Ng, C. Y., Yau, C. Y. \& Gao, J. Thickness dependence of superconductivity for $\mathrm{YBa}_{2} \mathrm{Cu}_{3} \mathrm{O}_{y}$ ultra-thin films. Supercond. Sci. Technol. 13, 580 (2000).
35. Lin, Z. et al. Quasi-two-dimensional superconductivity in $\mathrm{FeSe}_{0.3} T \mathrm{~T}_{0.7}$ thin films and electric-field modulation of superconducting transition. Sci. Rep. 5, 14133 (2015).

36. Werthamer, N. R., Helfand, E. \& Hohenberg, P. C. Temperature and purity dependence of the superconducting critical field, $H_{c 2}$. III. Electron spin and spin-orbit effects. Phys. Rev. 147, 295 (1966).

37. Khim, S. et al. Evidence for dominant Pauli paramagnetic effect in the upper critical field of single-crystalline $\mathrm{FeTe}_{0.6} \mathrm{Se}_{0.4}$. Phys. Rev. B 81, 184511 (2010).

38. Fuchs, G. et al. Orbital and spin effects for the upper critical field in As-deficient disordered Fe pnictide superconductors. New J. Phys. 11, 075007 (2009).

39. Savaloni, H., Khojier, K. \& Alaee, M. S. Characteristics of nanostructure and electrical properties of Ti thin films as a function of substrate temperature and film thickness. J. Mater. Sci. 42, 2603 (2007).

40. Kim, S. I. et al. Metallic conduction induced by direct anion site doping in layered SnSe. Sci. Rep. 6, 19733 (2016).

41. Xu, D., Yin, S., Zeng, X., Yang, S. \& Wen, X. Structural, optical and electrical properties of $\mathrm{ZnO}$ : B thin films with different thickness for bifacial a-Si:H/c-Si heterojunction solar cells. Front. Optoelectron. 10, 31 (2017).

42. Momin, A., Islam, M. A., Azad, M. A. A., Ali, M. \& Bhuiyan, M. R. A. Influence of the thickness on the electrical properties of CdS thin films. J. Appl. Sci. Tech. 7, 49 (2010).

43. He, J. et al. Electronic evidence of an insulator-superconductor crossover in single-layer $\mathrm{FeSe} / \mathrm{SrTiO}_{3}$ films. Proc. Natl. Acad. Sci. U.S.A. 111, 18501 (2014).

44. Graham, M. R., Adkins, C. J., Behar, H. \& Rosenbaum, R. Experimental study of the loffe-Regel criterion for amorphous indium oxide films. J. Phys.: Condens. Matter 10, 809 (1998).

45. Chand, $\mathrm{M}$. et al. Phase diagram of the strongly disordered s-wave superconductor NbN close to the metal-insulator transition. Phys. Rev. B 85, 014508 (2012).

46. Zhang, L. \& Tang, Z. J. Polaron relaxation and variable-range-hopping conductivity in the giant-dielectric-constant material $\mathrm{CaCu}_{3} \mathrm{Ti}_{4} \mathrm{O}_{12}$. Phys. Rev. B 70 174306 (2004).

47. Kovacs, I. A. \& Igloi, F. Renormalization group study of the two-dimensional random transverse-field Ising model. Phys. Rev. B 82, 054437 (2010).

48. Vojta, T., Farquhar, A. \& Mast, J. Infinite-randomness critical point in the twodimensional disordered contact process. Phys. Rev. E 79, 011111 (2009).

49. Theunissen, M. H. \& Kes, P. H. Resistive transitions of thin film superconductors in a magnetic field. Phys. Rev. B 55, 15183 (1997).

50. Qin, Y. G., Vicente, C. L. \& Yoon, J. Magnetically induced metallic phase in superconducting tantalum films. Phys. Rev. B 73, 100505 (2006). 